\title{
YAP mediates crosstalk between the Hippo and PI3K-TOR pathways by suppressing PTEN via miR-29
}

\author{
Karen Tumaneng ${ }^{1,2}$, Karin Schlegelmilch ${ }^{3,5}$, Ryan RusselI ${ }^{1}$, Dean Yimlamai ${ }^{3}$, Harihar \\ Basnet $^{2}$, Navin Mahadevan ${ }^{2}$, Julien Fitamant ${ }^{4}$, Nabeel Bardeesy ${ }^{4}$, Fernando Camargo ${ }^{3}$, and \\ Kun-Liang Guan ${ }^{1, *}$ \\ ${ }^{1}$ Department of Pharmacology and Moores Cancer Center, University of California at San Diego, \\ La Jolla, CA, 92093, USA \\ ${ }^{2}$ Biomedical Sciences Graduate Program, School of Medicine, University of California at San \\ Diego, La Jolla, CA, 92093, USA \\ ${ }^{3}$ Department of Stem Cell and Regenerative Biology, Harvard University, Cambridge, MA 02138, \\ USA \\ ${ }^{4}$ Department of Medicine, Harvard Medical School, Boston, MA 02114, USA \\ ${ }^{5}$ Institute for Chemistry/Biochemistry, FU Berlin, Berlin 14195, Germany
}

\section{Keywords}

Hippo; YAP; mTOR; PTEN; miR-29

\begin{abstract}
Organ development is a complex process governed by the interplay of several signaling pathways that play critical functions in the regulation of cell growth and proliferation. Over the past years, the Hippo pathway has emerged as a key regulator of organ size. Perturbation of this pathway has been shown to play important roles in tumorigenesis. YAP, the main downstream target of the mammalian Hippo pathway, promotes organ growth yet the underlying molecular mechanism of this regulation remains unclear. Here we provide evidence that YAP activates the mammalian target of rapamycin (mTOR), a major regulator of cell growth. We have identified the tumor suppressor PTEN, an upstream negative regulator of mTOR, as a critical mediator of YAP in mTOR regulation. We demonstrate that YAP downregulates PTEN by inducing miR-29 to inhibit PTEN translation. Lastly, we show that PI3K-mTOR is a pathway modulated by YAP to regulate cell size, tissue growth,
\end{abstract}

\footnotetext{
"Correspondence and requests for materials should be addressed to K.L.G. (kuguan@ucsd.edu). .

Supplementary Information

Supplementary information contains methods and supplementary figures/legends.

Author Contributions

K.T. performed the experiments. K.S. conducted the LY294002 animal experiment. K.T. and R.R. performed fluorescent IHC staining experiments. D.Y. prepared mouse tissue slides for IHC experiments. K.T. and H.B. performed luciferase and ChIP assays. K.T. and N.M. conducted flow cytometry experiments. J.F. and N.B. provided the Mst1/2 KO mouse liver tissues. K.S. and F.C. designed the LY294002 animal experiment. K.T. and K.L.G. designed experiments and wrote the manuscript.

Author Information

The authors declare no competing financial interests.
} 
and hyperplasia. Our studies reveal a functional link between Hippo and PI3K-mTOR, providing a molecular basis for the coordination of these two pathways in organ size regulation.

Hippo and TOR are two major signaling pathways involved in the regulation of organ size in Drosophila and mammals ${ }^{1-7}$. Genetic studies have established that the Hippo pathway plays a crucial role in organ size regulation by controlling cell number through modulation of cell proliferation and apoptosis ${ }^{8-11}$. A major downstream effector of the mammalian Hippo pathway is the Yes-associated protein (YAP) transcription co-activator, which is phosphorylated and inhibited by the Hippo pathway component Lats ${ }^{12,13}$. Acting upstream of Lats is Mst, which phosphorylates and activates Lats ${ }^{14}$. TOR is well established as a central pathway regulating cell growth (cell size) by integrating intracellular and extracellular signals to stimulate protein translation ${ }^{15}$. Because cell growth is required for proliferation, the function of the Hippo pathway is expected to be coordinated with TOR. Indeed, recent studies have provided evidence for a crosstalk between the Hippo and TOR pathways in Drosophila ${ }^{16,17}$. However, the underlying molecular mechanism of this crosstalk has not been elucidated.

We tested whether the canonical Hippo pathway affects mTOR signaling. We performed siRNA knockdown of Lats1/2 in MCF10A cells and found that Lats1/2 knockdown decreased YAP phosphorylation. Notably, Lats1/2 knockdown also resulted in the activation of mTORC1 and mTORC2, as evident in increased phosphorylation of S6K T389 and AKT S473, which are direct substrates of mTORC1 and mTORC2, respectively (Fig. 1a). Cell density is known to regulate YAP phosphorylation and activity ${ }^{7}$. Interestingly, high cell density also decreased phosphorylation of S6K and AKT along with elevated YAP phosphorylation (Fig. S1a). Subsequently, we examined the effects of YAP on mTORC1 and mTORC2 by generating stable MCF10A cells with YAP overexpression or YAP knockdown by shRNA. As shown in Fig. 1b, overexpression of YAP increased phosphorylation of both S6K T389 and AKT S473. Conversely, phosphorylation at these residues was decreased in YAP knockdown cells, indicating that YAP regulates mTOR activity. Similar effects of YAP on mTOR activity were observed in HeLa cells (Fig. S1b). We then investigated whether this regulation occurs in vivo using YAP transgenic mice ${ }^{18}$. Indeed, we observed an increase in S240/244 phosphorylation of S6 ribosomal protein, the substrate of S6K, in the intestinal crypt compartment, which was the region with the highest YAP expression (Fig. 1c), of the YAP transgenic mice. On the other hand, the crypt compartment of the control mice showed much lower p-S6 signal. Further, we observed an increase in S473 phosphorylation of AKT in the crypt compartment of the YAP transgenic mice (Fig. 1d). Together, these data support a role of YAP in mTORC1/2 regulation.

Several cues such as nutrients, serum, and growth factors can induce mTORC1 activation. We examined whether YAP depends on these factors to stimulate mTOR activity by performing serum starvation or amino acid starvation in control cells and YAPoverexpressing cells. Deprivation of serum or amino acid did not abolish the YAP-induced increase in S6K phosphorylation (Fig. S2a, S2b). YAP is known to induce expression of many genes, including growth factors ${ }^{19}$. To determine if the effect of YAP on mTORC1 activity was due to extrinsic factors released into the media, we performed a media swap 
experiment between control cells and YAP-overexpressing cells. Media swap did not affect S6K phosphorylation in the respective cells (Fig. S2c), indicating that the YAP-induced mTORC1 activation was not dependent on extrinsic factors. Consistently, no difference in ERK1/2 phosphorylation was observed between YAP overexpression and YAP knockdown cells (Fig. S2d).

To test whether YAP targets an already known component of the mTOR pathway, we examined the protein level of known upstream regulators of mTOR and of proteins that are in complex with mTOR. As shown in Fig. 2a, YAP overexpression or knockdown did not affect the protein expression of TSC2, AKT, mTOR, Raptor, and Rictor. However, YAP overexpression decreased the protein level of the tumor suppressor PTEN. Conversely, YAP knockdown increased PTEN protein level. This YAP-dependent regulation of PTEN was similarly observed in HeLa cells (Fig. S1b). Consistently, YAP-induced PTEN loss led to AKT activation, as indicated by increased phosphorylation of GSK3 and FoxO1, two wellcharacterized AKT substrates (Fig. 2b).

To determine whether PTEN was a critical target of YAP in activating mTOR, PTEN was ectopically expressed in YAP-overexpressing cells by transient transfection. In parallel, PTEN was knocked down in YAP knockdown cells. Indeed, re-expression of PTEN antagonized the effects of YAP on mTORC1 and mTORC2 (Fig. 2c). Conversely, knockdown of PTEN in YAP knockdown cells rescued mTORC1 and mTORC2 activities (Fig. 2d). Interestingly, in PTEN-null U87MG cells, YAP expression did not affect S6K phosphorylation (Fig. S2e). This YAP-dependent reduction of PTEN protein and the ability of PTEN to reverse the effects of YAP on mTORC1 and mTORC2 suggested that PTEN was a crucial mediator of YAP in the regulation of mTOR. Consistent with this, we found that inhibition of the PI3K-AKT pathway by Wortmannin abolished the YAP-induced activation of mTORC1 and mTORC2 (Fig. S2f). Further, we found that YAP overexpression in the intestine correlated with significant downregulation of PTEN (Fig. 2e, S2g).

Our lab has recently discovered that YAP phosphorylation is regulated by G-protein-coupled receptor (GPCR) signaling ${ }^{20}$. For example, thrombin, a ligand for the GPCR PAR1, induces YAP activation in MCF10A cells ${ }^{21}$. Therefore, we used thrombin as a stimulus to determine the physiological function of YAP in PTEN regulation. We found that thrombin treatment in MCF10A cells resulted in YAP activation and PTEN downregulation (Fig. 2f). In the absence of YAP, the effect of thrombin on PTEN was abolished. Collectively, these data establish a function of YAP in regulating PTEN.

Previously, it has been reported that liver-specific knockout of Mst1/2 increases S6K phosphorylation $^{22}$. We therefore examined the status of PTEN in Mst $1 / 2^{-/-}$mice. Interestingly, we found that PTEN expression was significantly reduced in the livers of Mst1/2- ${ }^{-/}$mice and this correlated with YAP activation/dephosphorylation (Fig. $2 \mathrm{~g}$ ), reinforcing a crosstalk between Hippo and PI3K-TOR via regulation of PTEN by YAP.

We sought to determine the mechanism through which YAP suppresses PTEN. As a transcription co-activator, YAP is known to induce gene transcription ${ }^{23}$. We examined the 
effect of YAP on PTEN transcription by qRT-PCR and found that YAP overexpression or knockdown did not significantly alter the level of PTEN mRNA (Fig. S3a). Subsequently, we examined the effects of YAP on PTEN protein stability and degradation by performing time-course experiments with cycloheximide and MG132, respectively. However, we did not observe a significant difference in the expression of PTEN among the stable cells (Fig. S3b, S3c). Consistently, YAP did not affect PTEN phosphorylation at T380/S382/S383 (Fig. S3d), which has been reported to regulate PTEN protein stability ${ }^{24}$.

Because YAP affected neither PTEN transcription nor protein stability, we hypothesized that YAP may regulate PTEN translation possibly through microRNAs. MicroRNAs are important regulators of gene expression by inhibiting translation of their target mRNAs ${ }^{25}$. Previously, it has been reported that the Drosophila homolog of YAP, Yorkie, induces the expression of the bantam microRNA that serves as a critical mediator of Yorkie's biological functions $^{26,27}$. Given the high conservation of the Hippo pathway, we investigated whether the YAP-induced PTEN reduction was mediated by microRNAs. First, we knocked down Dicer by siRNA in YAP-overexpressing cells. Interestingly, we found that Dicer knockdown restored PTEN expression in YAP-overexpressing cells (Fig. 3a), suggesting that microRNAs are important for PTEN regulation by YAP. To determine whether YAP induces the expression of microRNAs, we used genome-wide deep sequencing technology to identify potential microRNAs induced by YAP. Our data showed that YAP overexpression resulted in the upregulation of microRNAs, particularly the miR-29a/b/c family (Table 1, see Accession number in Methods). Conversely, YAP knockdown resulted in the downregulation of miR-29a/b/c. The regulation of miR-29 expression by YAP was verified by qRT-PCR (Fig. 3b). We also found that the levels of miR-29a/b/c were upregulated by thrombin (Fig. 3c). Interestingly, thrombin downregulates the expression of these microRNAs in YAP knockdown cells. Together, these data suggest a function of YAP in the regulation of miR-29.

Interestingly, the miR-29 family is predicted to target PTEN, as indicated by the presence of the miR-29a/b/c consensus site in the $3^{\prime}$ untranslated region (UTR) of PTEN (Fig. S3e). Recently, two studies have confirmed that miR-29a and miR-29b target PTEN to regulate cell migration ${ }^{28,29}$. To determine whether miR-29c also regulates PTEN translation by targeting the $3^{\prime}$ UTR of PTEN, we performed a luciferase assay with control miR or miR-29c. As shown in Fig. 3c, miR-29c reduced a luciferase reporter that had the luciferase coding sequence fused with the PTEN 3'UTR but did not affect the control reporter without the PTEN 3'UTR. Moreover, when the putative binding sites for miR-29c were deleted, the miR-29c-induced decrease in luciferase reading was abolished (Fig. 3d). Subsequently, we determined whether miR-29c regulates the PTEN protein by transiently inducing or inhibiting miR-29c in MCF10A cells. Transient overexpression or inhibition of miR-29c resulted in noticeable changes in PTEN protein levels (Fig. 3e). Together, these data suggest that miR-29c downregulates PTEN protein by targeting the PTEN $3{ }^{\prime}$ UTR.

We then investigated whether miR-29c is a direct target of YAP. Sequence analysis of the promoter region of miR-29c reveals putative binding sites for the TEAD transcription factors (Fig. S3f), the major transcription factors mediating YAP functions ${ }^{30,} 31$. We performed chromatin immunoprecipitation (ChIP) with YAP antibody, followed by qPCR 
using primers for the miR-29c promoter. Our data showed that YAP did bind the promoter region of miR-29c, particularly the region 150-300bp upstream of the transcription start site that harbors a TEAD consensus site (Fig. 3f). CTGF, a known YAP-TEAD target gene, and GAPDH were used as positive and negative controls, respectively. Thus, YAP appears to directly induce miR-29c to target PTEN. The promoters of miR-29a and miR-29b1/2 also contain binding sites for TEADs (Fig. S3f), suggesting that YAP transcriptionally regulates miR-29 to inhibit PTEN. Consistent with our observations, an inverse correlation between PTEN and miR-29 has been reported in hepatocellular carcinoma ${ }^{29}$.

To confirm the role of miR-29 in the regulation of PTEN by YAP, we transiently overexpressed each miR-29 in YAP knockdown cells and in parallel transiently inhibited each miR-29 in YAP-overexpressing cells. We found that manipulation of a single miR-29 was sufficient to alter the effects of YAP overexpression or YAP knockdown on PTEN (Fig. $3 \mathrm{~g}$ ). Furthermore, each miR-29 is sufficient to rescue the effects of YAP knockdown on phosphorylation of S6K and S6 (Fig. 3h). These data support that the miR-29 family is important for the regulation of PTEN by YAP.

We investigated the physiological relevance of YAP-induced mTOR activation via PTEN. We observed that YAP overexpression increased cyclin D1 expression (Fig. S4a).

Subsequently, we examined whether YAP regulates cell size. We cultured stable MCF10A cells to $\sim 40 \%$ confluence, then performed overnight serum starvation to synchronize cells, and analyzed cell size by flow cytometry using forward scatter. Our data showed that cell size was increased in YAP-overexpressing cells and more significantly in the constitutively active YAP-5SA-expressing cells (Fig. 4a). Interestingly, the YAP-induced increase in cell size was mimicked by PTEN knockdown (Fig. 4b). To determine whether mTOR mediates YAP-induced increase in cell size, we inhibited mTOR activity by rapamycin in control cells and YAP-overexpressing cells. We found that rapamycin treatment indeed abolished the YAP-induced increase in cell size, and the corresponding histogram of YAPoverexpressing cells with rapamycin treatment overlapped with that of control cells with rapamycin treatment (Fig. 4c).

Similarly, treatment with LY294002, which also inhibits mTOR via inhibition of PI3K, abolished the effect of YAP on cell size (Fig. 4d). We also determined whether YAP regulates cell size in vivo using transgenic mice with YAP overexpression in the $\operatorname{skin}^{32}$. We found that the size of keratinocytes in the YAP transgenic mice was significantly larger compared to that in control mice (Fig. 4e). Together, these data demonstrate a function of YAP in cell size regulation in a manner dependent on PI3K-mTOR. Although transient overexpression of miR-29 positively contributes to mTOR activity (Fig. 3h), we did not observe a detectable effect of miR-29 on cell size, possibly due to a weak activation of mTOR from transient overexpression of miR-29.

Lastly, we determined the functional significance of the Hippo-PI3K-mTOR crosstalk in vivo. It has been reported that YAP overexpression in the skin leads to tissue expansion and hyperplasia ${ }^{32}$. Notably, we found that YAP overexpression in the skin resulted in mTOR activation (Fig. 4f). Interestingly, we also observed a downregulation of the PTEN protein in the YAP transgenic mice (Fig. 4g). Therefore, we performed LY294002 treatment in control 
mice and YAP transgenic mice. As shown in Fig. 4f, activation of YAP under the K14-Cre promoter resulted in thickening and hyperplasia of the epidermis and the tongue. The extent of hyperplasia was reduced upon inhibition of the PI3K pathway in the YAP transgenic mice (Fig. 4h), suggesting that the PTEN axis is an important component contributing to YAPdriven tissue overgrowth in vivo. Consistently, decreased phosphorylation of S6 confirmed the inhibition of mTOR in the YAP transgenic mice by LY294002 (Fig. 4i), and correlated with reduction of YAP-induced overgrowth and hyperplasia (Fig. 4h). These findings suggest that PI3K-mTOR is a pathway modulated by YAP to drive tissue expansion.

In summary, our studies establish a functional link between Hippo and TOR, the two major regulators of organ size. Coordination between these two pathways is expected given the function of YAP in cell proliferation, which cannot be sustained without cell growth. This report provides a molecular mechanism through which the Hippo pathway regulates cell growth via TOR. YAP mediates the major effects of the Hippo pathway by regulating gene expression and among the YAP target genes is the miR-29 family, which inhibits PTEN by targeting its 3'UTR. PTEN is a key antagonist of PI3K, which is well established as an upstream activator of mTOR. Hence, the Hippo pathway inhibits cell growth by inactivating the mTOR pathway (both mTORC1 and mTORC2), and this is executed at least in part through the actions of YAP, miR-29, and PTEN, subsequently affecting AKT, TSC1/2, and Rheb (Fig. S5). When organ growth is needed the Hippo pathway will be inactivated, which leads to YAP activation and gene expression-such as miR-29 to promote cell growth and cyclin D1 to induce proliferation-resulting in the eventual increase in organ size.

The functional regulation of PTEN by YAP revealed in this study has important implications in growth control and cancer biology. PTEN is a key negative regulator of the cell-survival signaling pathway initiated by PI3K, whose activation is triggered by ligand binding to receptor tyrosine kinases (RTKs). mTOR functions as a nutrient sensor that integrates PI3Kmediated growth factor signaling to regulate cell growth. The recent discovery of the Hippo pathway as a downstream signaling branch of GPCRs suggests that a wide range of secreted signals could modulate growth through the Hippo pathway. The link from YAP to PTEN to mTOR reveals a possible integration of GPCRs, RTKs, and nutrient status in growth control, illustrating how a web of signaling networks coordinate with each other to fine-tune physiological and pathological processes. Given the prominent roles of both YAP and PTEN in tumorigenesis, it would be interesting to explore the interplay of these genes in human cancers.

\section{Methods}

Methods described in Supplementary Information.

\section{Supplementary Material}

Refer to Web version on PubMed Central for supplementary material. 


\section{Acknowledgments}

We thank Dr. Frank Furnari and Dr. Max Wicha for reagents. We thank Dr. Jiagang Zhao for technical help, and Dr. Joungmok Kim and Dr. Bin Zhao for thoughtful discussions. Deep sequencing service was provided by LC Sciences. K.T. was supported in part by the UCSD Graduate Training Program in Cellular and Molecular Pharmacology. K.L.G. is supported by grants from NIH.

\section{References}

1. Dong J, et al. Elucidation of a universal size-control mechanism in Drosophila and mammals. Cell. 2007; 130:1120-1133. [PubMed: 17889654]

2. Lee KP, et al. The Hippo-Salvador pathway restrains hepatic oval cell proliferation, liver size, and liver tumorigenesis. Proceedings of the National Academy of Sciences of the United States of America. 2010; 107:8248-8253. [PubMed: 20404163]

3. Lu L, et al. Hippo signaling is a potent in vivo growth and tumor suppressor pathway in the mammalian liver. Proceedings of the National Academy of Sciences of the United States of America. 2010; 107:1437-1442. [PubMed: 20080689]

4. Shima $\mathrm{H}$, et al. Disruption of the $\mathrm{p} 70(\mathrm{~s} 6 \mathrm{k}) / \mathrm{p} 85(\mathrm{~s} 6 \mathrm{k})$ gene reveals a small mouse phenotype and a new functional S6 kinase. The EMBO journal. 1998; 17:6649-6659. [PubMed: 9822608]

5. Tamemoto $\mathrm{H}$, et al. Insulin resistance and growth retardation in mice lacking insulin receptor substrate-1. Nature. 1994; 372:182-186. [PubMed: 7969452]

6. Verdu J, Buratovich MA, Wilder EL, Birnbaum MJ. Cell-autonomous regulation of cell and organ growth in Drosophila by Akt/PKB. Nature cell biology. 1999; 1:500-506.

7. Zhao B, et al. Inactivation of YAP oncoprotein by the Hippo pathway is involved in cell contact inhibition and tissue growth control. Genes \& development. 2007; 21:2747-2761. [PubMed: 17974916]

8. Hamaratoglu F, et al. The tumour-suppressor genes NF2/Merlin and Expanded act through Hippo signalling to regulate cell proliferation and apoptosis. Nature cell biology. 2006; 8:27-36.

9. Huang J, Wu S, Barrera J, Matthews K, Pan D. The Hippo signaling pathway coordinately regulates cell proliferation and apoptosis by inactivating Yorkie, the Drosophila Homolog of YAP. Cell. 2005; 122:421-434. [PubMed: 16096061]

10. Lai ZC, et al. Control of cell proliferation and apoptosis by mob as tumor suppressor, mats. Cell. 2005; 120:675-685. [PubMed: 15766530]

11. Wu S, Huang J, Dong J, Pan D. hippo encodes a Ste-20 family protein kinase that restricts cell proliferation and promotes apoptosis in conjunction with salvador and warts. Cell. 2003; 114:445456. [PubMed: 12941273]

12. Hao Y, Chun A, Cheung K, Rashidi B, Yang X. Tumor suppressor LATS1 is a negative regulator of oncogene YAP. The Journal of biological chemistry. 2008; 283:5496-5509. [PubMed: 18158288]

13. Zhang J, Smolen GA, Haber DA. Negative regulation of YAP by LATS1 underscores evolutionary conservation of the Drosophila Hippo pathway. Cancer research. 2008; 68:2789-2794. [PubMed: 18413746]

14. Chan EH, et al. The Ste20-like kinase Mst2 activates the human large tumor suppressor kinase Lats1. Oncogene. 2005; 24:2076-2086. [PubMed: 15688006]

15. Kim DH, et al. mTOR interacts with raptor to form a nutrient-sensitive complex that signals to the cell growth machinery. Cell. 2002; 110:163-175. [PubMed: 12150925]

16. Strassburger K, Tiebe M, Pinna F, Breuhahn K, Teleman AA. Insulin/IGF signaling drives cell proliferation in part via Yorkie/YAP. Developmental biology. 2012; 367:187-196. [PubMed: 22609549]

17. Ye X, Deng Y, Lai ZC. Akt is negatively regulated by Hippo signaling for growth inhibition in Drosophila. Developmental biology. 2012; 369:115-123. [PubMed: 22732571]

18. Camargo FD, et al. YAP1 increases organ size and expands undifferentiated progenitor cells. Current biology: CB. 2007; 17:2054-2060. [PubMed: 17980593] 
19. Zhang J, et al. YAP-dependent induction of amphiregulin identifies a non-cell-autonomous component of the Hippo pathway. Nature cell biology. 2009; 11:1444-1450.

20. Yu FX, et al. Regulation of the Hippo-YAP Pathway by G-Protein-Coupled Receptor Signaling. Cell. 2012; 150:780-791. [PubMed: 22863277]

21. Mo JS, Yu FX, Gong R, Brown JH, Guan KLG. Regulation of the Hippo-YAP pathway by protease-activated receptors (PARs). Genes \& development. 2012; 26

22. Zhou D, et al. Mst1 and Mst2 maintain hepatocyte quiescence and suppress hepatocellular carcinoma development through inactivation of the Yap1 oncogene. Cancer cell. 2009; 16:425438. [PubMed: 19878874]

23. Yagi R, Chen LF, Shigesada K, Murakami Y, Ito Y. A WW domain-containing yes-associated protein (YAP) is a novel transcriptional co-activator. The EMBO journal. 1999; 18:2551-2562. [PubMed: 10228168]

24. Vazquez F, Ramaswamy S, Nakamura N, Sellers WR. Phosphorylation of the PTEN tail regulates protein stability and function. Molecular and cellular biology. 2000; 20:5010-5018. [PubMed: 10866658]

25. Fabian MR, Sonenberg N, Filipowicz W. Regulation of mRNA translation and stability by microRNAs. Annual review of biochemistry. 2010; 79:351-379.

26. Thompson BJ, Cohen SM. The Hippo pathway regulates the bantam microRNA to control cell proliferation and apoptosis in Drosophila. Cell. 2006; 126:767-774. [PubMed: 16923395]

27. Nolo R, Morrison CM, Tao C, Zhang X, Halder G. The bantam microRNA is a target of the hippo tumor-suppressor pathway. Current biology: CB. 2006; 16:1895-1904. [PubMed: 16949821]

28. Wang C, Bian Z, Wei D, Zhang JG. miR-29b regulates migration of human breast cancer cells. Molecular and cellular biochemistry. 2011; 352:197-207. [PubMed: 21359530]

29. Kong G, et al. Upregulated microRNA-29a by hepatitis B virus X protein enhances hepatoma cell migration by targeting PTEN in cell culture model. PloS one. 2011; 6:e19518. [PubMed: 21573166]

30. Zhao B, et al. TEAD mediates YAP-dependent gene induction and growth control. Genes \& development. 2008; 22:1962-1971. [PubMed: 18579750]

31. Cao X, Pfaff SL, Gage FH. YAP regulates neural progenitor cell number via the TEA domain transcription factor. Genes \& development. 2008; 22:3320-3334. [PubMed: 19015275]

32. Schlegelmilch K, et al. Yap1 acts downstream of alpha-catenin to control epidermal proliferation. Cell. 2011; 144:782-795. [PubMed: 21376238] 
a

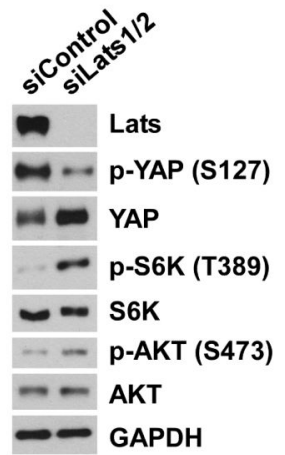

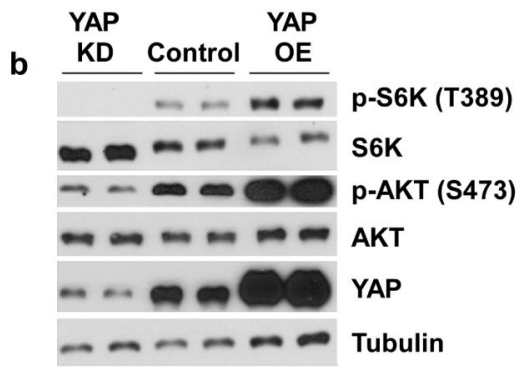
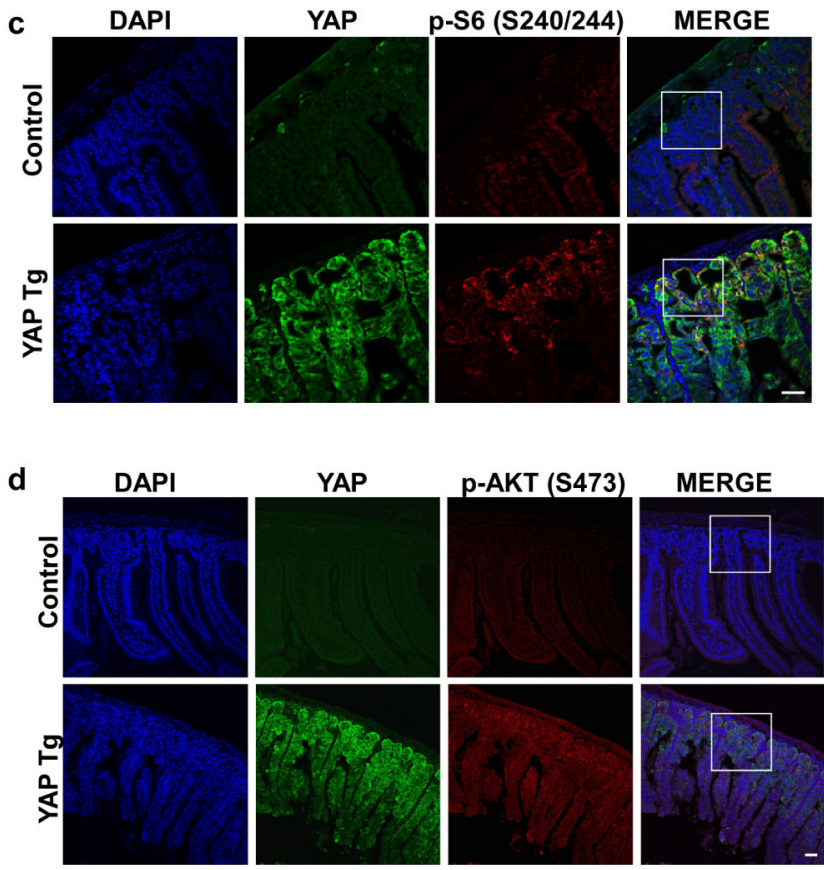

MERGE
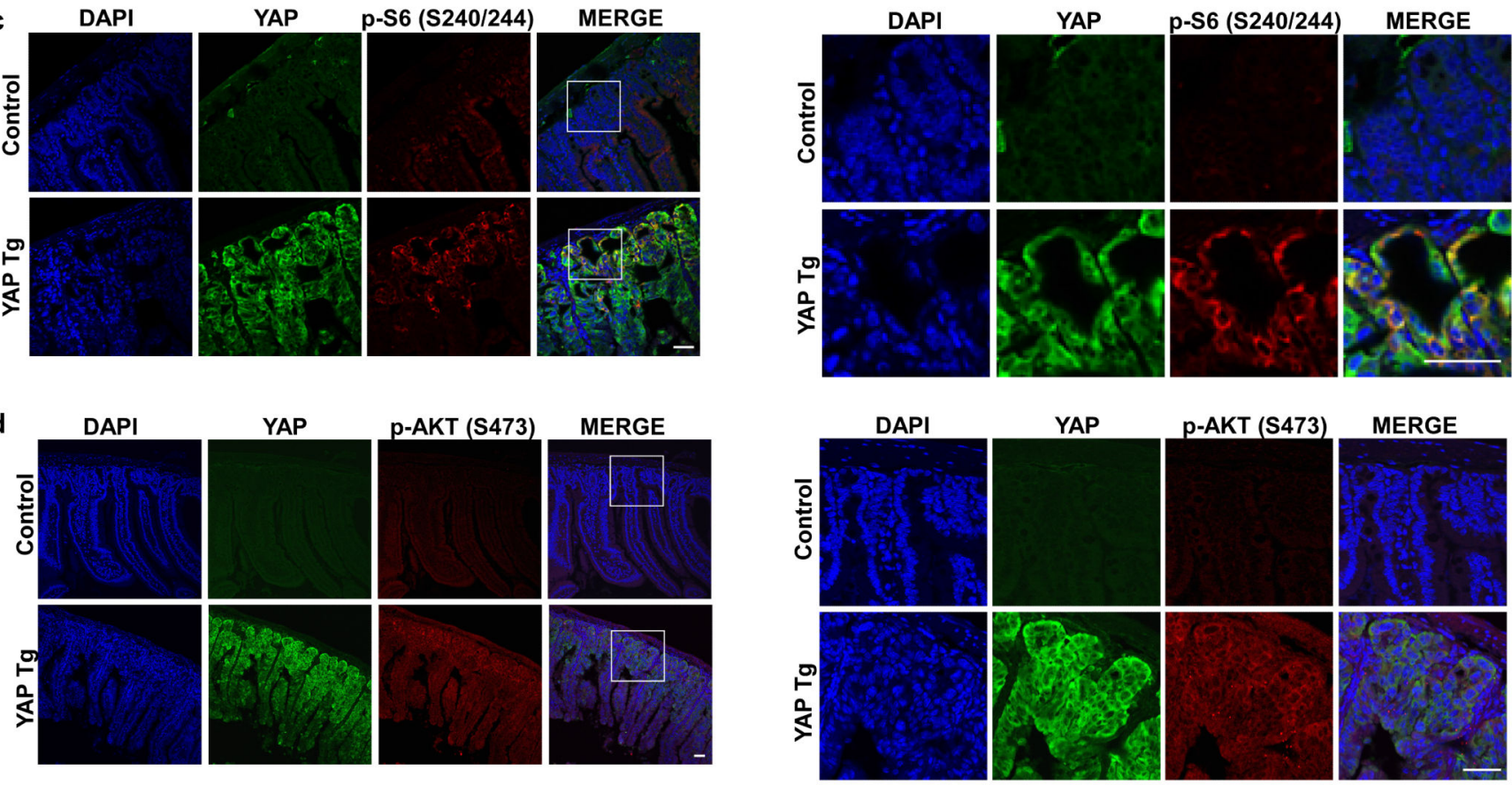

Figure 1. YAP regulates mTOR activity

a, Lats1/2 knockdown results in mTORC1/2 activation. MCF10A cells were transfected with non-targeting siRNA or siRNA targeting Lats1/2. Lysates were collected after 3 days for immunoblotting. b, YAP expression regulates mTORC1/2 activity. Stable MCF10A cell lines with YAP overexpression, YAP knockdown by shRNA, or control vector were generated. Cell lysates were collected and immunoblotted with the indicated antibodies. c, d, YAP overexpression activates mTORC1/2 in vivo. Immunohistochemical staining was performed on paraffin-embedded intestine tissues from control mice and YAP transgenic mice. Staining of YAP (Green), p-S6 or p-AKT (Red), and DAPI (Blue) is shown (Scale bar, $40 \mu \mathrm{m})$. Shown on the right are magnifications of the indicated boxed regions. 
a
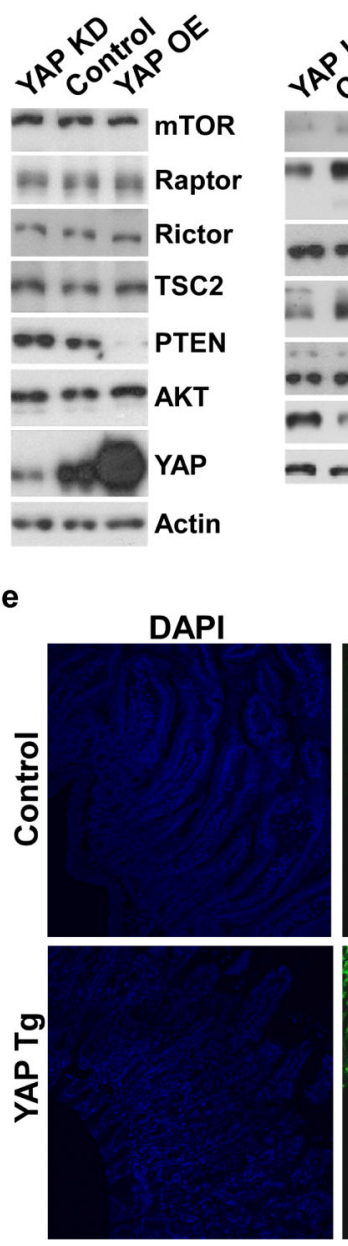

f

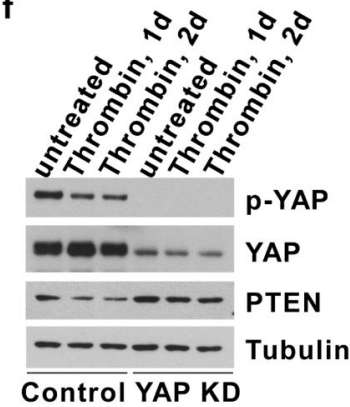

b

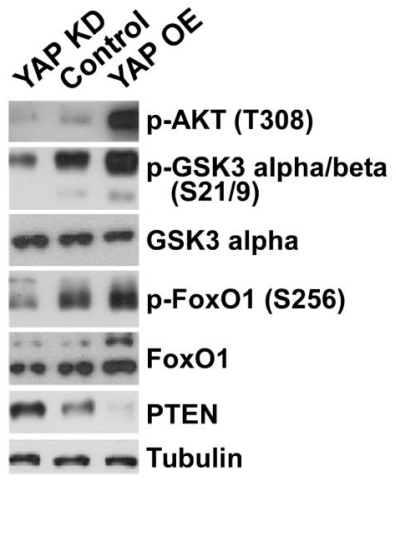

C

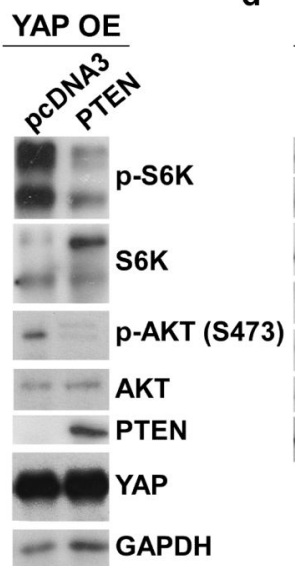

d
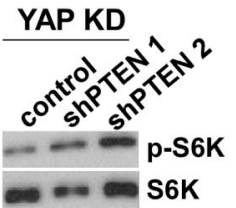

- - p-AKT (S473)
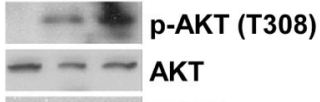

PTEN

$-\infty$ GAPDH
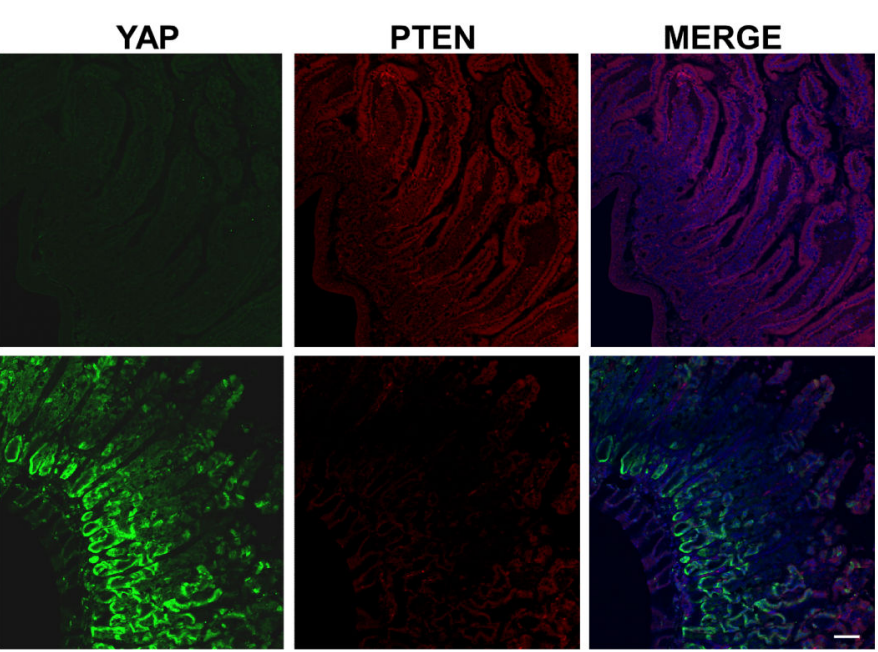

g

Figure 2. PTEN is a critical mediator of YAP in the regulation of $\mathrm{mTORC1/2}$ activity a, YAP expression regulates PTEN protein level. Lysates from the indicated stable cells were collected at $\sim 60-70 \%$ cell confluence for immunoblotting. $\mathbf{b}$, YAP-induced PTEN loss leads to increased AKT signaling. Experiment was performed similarly as in (a). c, Reexpression of PTEN suppresses the effects of YAP on mTORC1/2 activity a. Stable YAPoverexpressing MCF10A cell line was transfected with pcDNA3 or plasmid encoding PTEN. Three days later, lysates were collected for immunoblotting. c, PTEN knockdown rescues mTORC1/2 activity in YAP knockdown cells. Stable YAP knockdown MCF10A 
cell line was used as a background cell line to generate stable cells with control or shRNA for PTEN (shPTEN 1and shPTEN 2). e, YAP overexpression downregulates PTEN expression in vivo. Immunohistochemical staining was performed on paraffin-embedded intestine tissues from control mice and YAP transgenic mice using anti-YAP (Green) and anti-PTEN (Red) antibodies (Scale bar, $40 \mu \mathrm{m}$ ). f, Thrombin activates YAP and downregulates PTEN expression in a manner dependent on YAP. Stable MC10A cells with control vector or YAP knockdown were serum-starved for $24 \mathrm{~h}$, and then cultured for the indicated times in the presence or absence of thrombin $(10 \mathrm{U} / \mathrm{mL})$. g, Mst1/2 knockout results in PTEN downregulation. Lysates were prepared from liver tissues of wild-type mice and $\mathrm{Mst} 1 / 2^{-/-}$mice (3 mice per group), and immunoblotted using the indicated antibodies. 
a

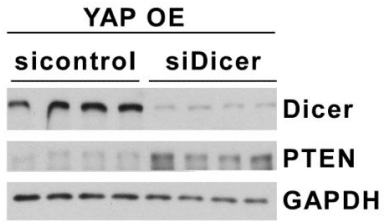

C

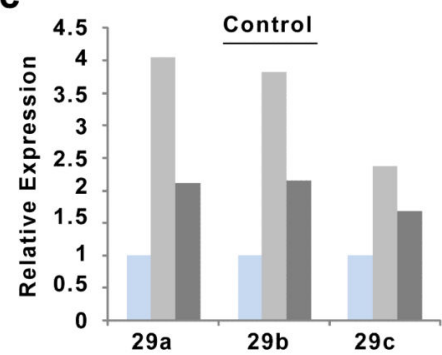

b
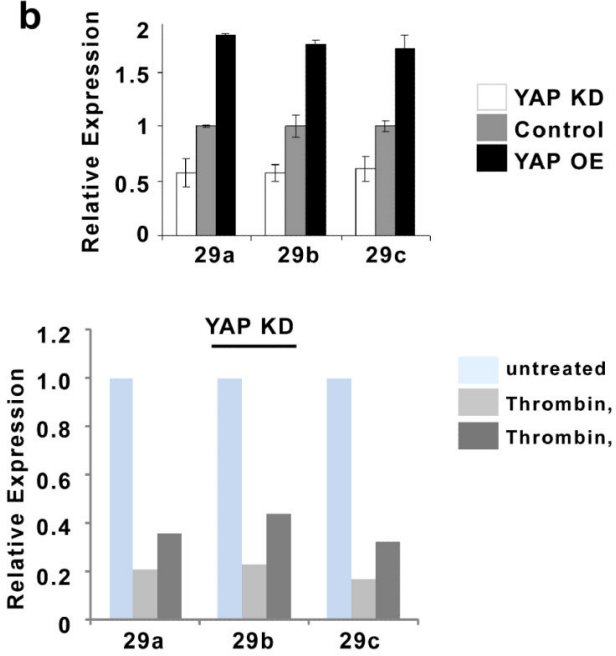

e

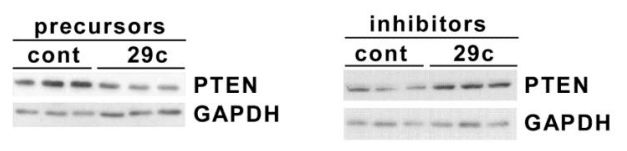

d

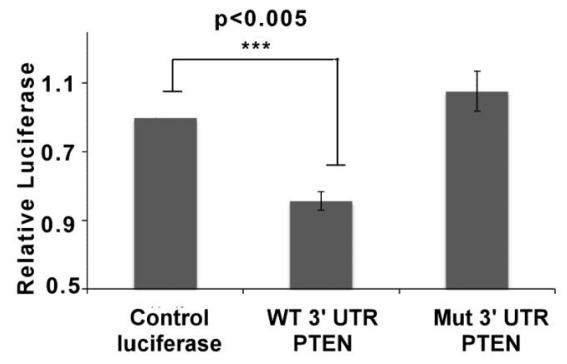

YAP KD

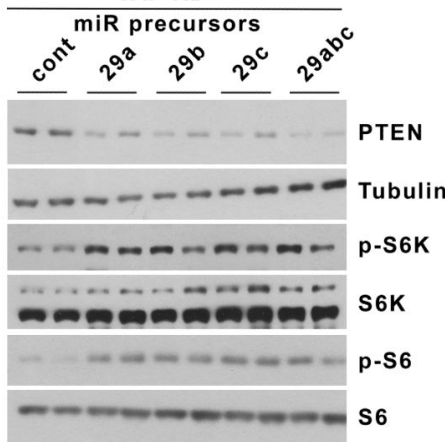

Figure 3. YAP directly induces the expression of miR-29c to target PTEN

a, YAP-induced PTEN loss is mediated by microRNAs. Stable MCF10A cell line with YAP overexpression was transfected with non-targeting siRNA or siRNA targeting Dicer. b, YAP regulates the expression of the miR-29 family. miRNA expression was validated by Taqman microRNA assay ( $n=3$, mean \pm s.e.m.). Data were obtained from three independent experiments. c, Thrombin induces miR-29 expression in a YAP-dependent manner. Stable MC10A cells with control vector or YAP overexpression were serum-starved for $24 \mathrm{~h}$, and then cultured for the indicated times in the presence or absence of thrombin $(10 \mathrm{U} / \mathrm{mL})$. Data 
were obtained from two independent experiments. d, miR-29c regulates the $3^{\prime}$ UTR of PTEN. HEK293 cells were transfected with control miR or miR-29c, and the indicated luciferase constructs. Luciferase activities were assayed $48 \mathrm{hr}$ after transfection and normalized first to control miR and then to control luciferase ( $\mathrm{n}=3$, mean \pm s.e.m.). Data were obtained from three independent experiments. e, miR-29c regulates PTEN protein level. MCF10A cells were transiently transfected with precursor or inhibitor for miR-29c. Cell medium was changed the following day and lysates were collected four days later for immunoblotting. f, YAP binds the promoter of miR-29c. Chromatin immunoprecipitation was performed using anti-YAP and anti-IgG antibodies, followed by quantitative PCR using primers specific to the indicated promoter regions ( $n=3$, mean \pm s.e.m.). Data were obtained from three independent experiments. g, miR-29 mediates the effects of YAP on PTEN protein. Precursors and inhibitors for $\mathrm{miR}-29 \mathrm{a} / \mathrm{b} / \mathrm{c}$ were transiently transfected in the indicated stable cells. Cell medium was changed the following day, and cell lysates were harvested after four days. h, Expression of a miR-29 is sufficient to rescue the effects of YAP knockdown on S6K phosphorylation. Experiment was performed similarly as in (g) and lysates were harvested six days later. 
a

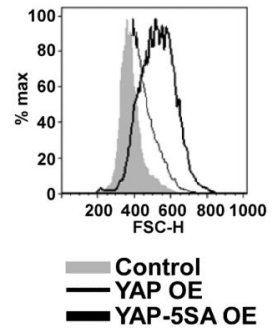

b

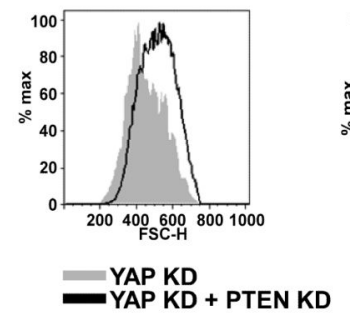

C

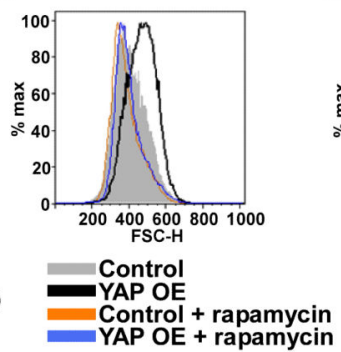

d

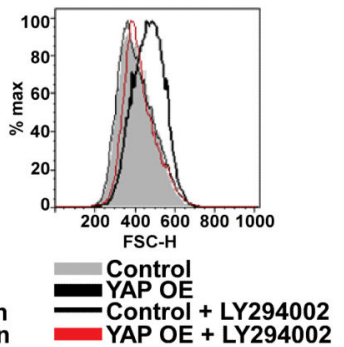

e

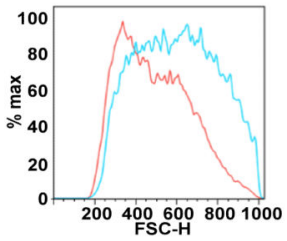

Control

f

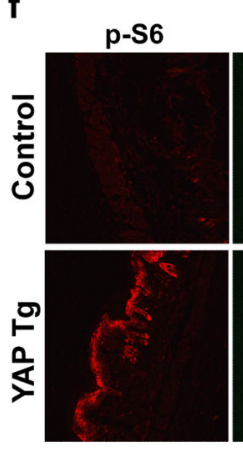

YAP

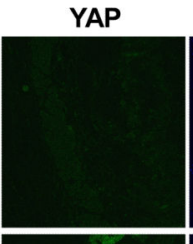

DAPI
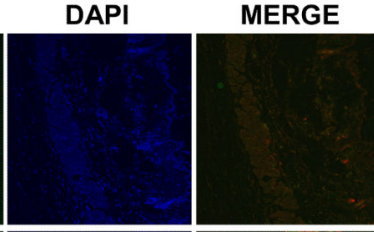

g PTEN
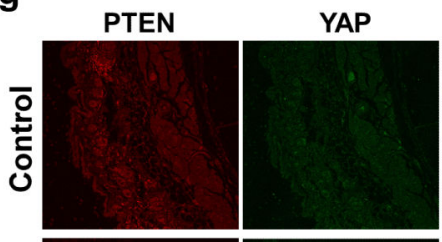

DAPI
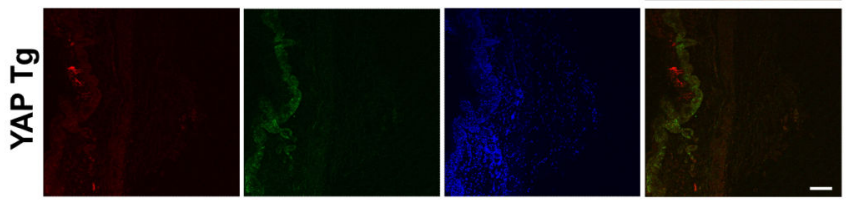

h

H\&E: Tongue Control-Vehicle YAP OE-Vehicle
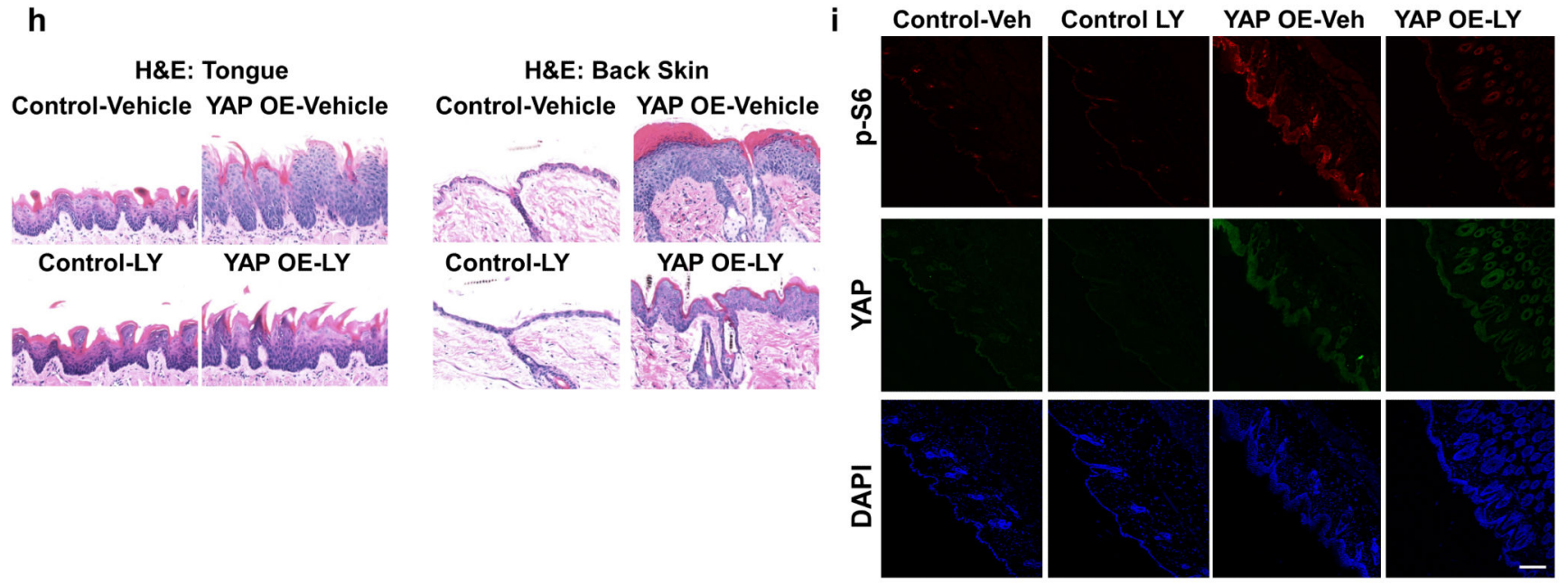

Figure 4. YAP regulates cell size and tissue growth via modulation of PI3K and mTOR a-e, YAP increases cell size in a manner dependent on the PI3K/PTEN-mTOR pathway. Flow cytometry was performed using forward scatter. a, Stable MCF10A cell lines with control vector, YAP overexpression, or constitutively active YAP overexpression were grown to $\sim 40 \%$ confluence and serum-starved overnight, then trypsinized, washed with $0.5 \%$ BSA/PBS, and stained with 7-AAD to exclude dead cells. b, Stable YAP knockdown MCF10A cell line was used as a background to knockdown PTEN. Cells with both YAP and PTEN knockdown were puromycin-resistant and dsRed ${ }^{+}$whereas cells with only YAP knockdown were puromycin-resistant and dsRed ${ }^{-}$. Cells were serum-starved overnight. c, d, Cells were grown to $\sim 30 \%$ confluence, then treated with rapamycin (20 nM), LY294002 (10 $\mu \mathrm{M})$ for $36 \mathrm{hr}$, then serum-starved overnight. e, Keratinocytes were isolated from the skin tissues of control mice and YAPtransgenic mice. $\mathbf{f}-\mathbf{g}$, Activation of YAP in the skin results 
in increased mTORC1 activity and decreased PTEN expression. Immunohistochemical staining was performed on paraffin-embedded skin tissues from control mice and YAP transgenic mice. Staining of YAP (Green), p-S6 or PTEN (Red), and DAPI (Blue) is shown (Scale bar, $40 \mu \mathrm{m}$ ). h, Inhibition of the PI3K-mTOR pathway diminishes YAP-induced hyperplasia. H\&E staining was performed using skin and tongue tissues from the indicated mice. LY denotes injection of LY294002. i, Reduced p-S6 staining confirms reduction of PI3K-mTOR activity by LY294002. Staining of YAP (Green), p-S6 (Red), and DAPI (Blue) is shown (Scale bar, $100 \mu \mathrm{m}$ ). 


\section{Table 1}

YAP regulates the expression of miR-29 family. Deep sequencing was performed using miRNA-enriched RNA from stable MCF10A cells with YAP overexpression, YAP knockdown, or control vector. miRNAs that display high expression in YAP-overexpressing cells and low expression in YAP knockdown cells are listed.

\begin{tabular}{lrrrrrr}
\hline & \multicolumn{3}{c}{ Normalized Copy \# } & \multicolumn{3}{c}{ Ratio of Normalized Copy \# } \\
miR_name & YAP KD & control & YAP OE & YAP KD:control & YAP OE:control & YAP KD: YAP OE \\
miR-152 & 16 & 48 & 200 & 0.32 & 4.13 & 0.08 \\
miR-1261 & 20 & 89 & 106 & 0.23 & 1.20 & 0.19 \\
miR-33a & 5 & 13 & 20 & 0.38 & 1.61 & 0.24 \\
miR-130a & 161 & 368 & 667 & 0.44 & 1.81 & 0.24 \\
miR-138 & 7 & 17 & 24 & 0.42 & 1.42 & 0.30 \\
miR-29a & 38,806 & 74,371 & 130,163 & 0.52 & 1.75 & 0.30 \\
miR-30c & 8 & 10 & 26 & 0.85 & 2.66 & 0.32 \\
miR-28 & 30 & 66 & 80 & 0.45 & 1.32 & 0.35 \\
miR-148a & 114 & 221 & 300 & 0.51 & 1.36 & 0.38 \\
miR-29b & 884 & 1,379 & 2,030 & 0.64 & 1.47 & 0.44 \\
miR-29c & 1,215 & 1,533 & 2,429 & 0.79 & 1.58 & 0.50 \\
\hline
\end{tabular}

